JOURNAL OF THE

AMERICAN MATHEMATICAL SOCIETY

Volume 12, Number 1, January 1999, Pages 85-92

S 0894-0347(99)00285-4

\title{
DEFORMATIONS OF CANONICAL SINGULARITIES
}

\author{
YUJIRO KAWAMATA
}

\section{INTRODUCTION}

M. Reid defined the concept of canonical singularities as those which appear on canonical models of algebraic varieties whose canonical rings are finitely generated. From the view point of the classification theory of algebraic varieties, it is expected that small deformations of a variety which has only canonical singularities have only canonical singularities. The main result of this paper states that this is the case:

Main Theorem. Let $\pi: X \rightarrow S$ be a flat morphism from a germ of a variety $\left(X, x_{0}\right)$ to a germ of a smooth curve $\left(S, s_{0}\right)$ whose special fiber $X_{0}=\pi^{-1}\left(s_{0}\right)$ has only canonical singularities. Then $X$ has only canonical singularities. In particular, the fibers $X_{s}=\pi^{-1}(s)$ have only canonical singularities.

A normal variety $X$ is said to have only canonical singularities if the canonical divisor $K_{X}$ is a $\mathbb{Q}$-Cartier divisor and if the discrepancy divisor $K_{Y}-\mu^{*} K_{X}$ is effective for a resolution of singularities $\mu: Y \rightarrow X$ (cf. [KMM]). For example, 2-dimensional canonical singularities are nothing but Du Val (or rational double) singularities. It is well known that deformations of $\mathrm{Du}$ Val singularities are Du Val singularities. Our theorem was not known even for the 3-dimensional case. A similar problem for terminal singularities is still open. A result has already been obtained for log terminal singularities $([\mathrm{I}])$.

The proof of the Main Theorem follows closely the argument of Siu [Si] who proved the following: if $\pi: X \rightarrow S$ is a smooth projective family of varieties over a base space such that all the fibers $X_{s}$ are of general type, then the plurigenus $P_{m}\left(X_{s}\right)$ of the fibers is constant for each positive integer $m$. The difference is that our method is algebraic instead of analytic. We can also adapt the analytic proof of Siu's theorem to the algebraic one by using our method (Theorem 6).

The concept of multiplier ideal sheaf introduced by Nadel in the analytic setting plays an important role in the proof. The point is that the multiplier ideal sheaf has a canonical embedding to the structure sheaf, and one can consider it independently from the original linear system. In order to deal with an infinite sequence of linear systems, we consider an increasing sequence of multiplier ideal sheaves instead of considering the infinite sum of metrics as in [Si].

Received by the editors January 31, 1998.

1991 Mathematics Subject Classification. Primary 14B07, 14E30, 14 F17.

Key words and phrases. Canonical singularity, deformation.

(C)1999 American Mathematical Society 


\section{Proof of the Main Theorem}

Let $\mu: Y \rightarrow X$ be a projective birational morphism from a smooth variety such that $Y_{0}=\mu^{*} X_{0}=X_{0}^{\prime}+E$ has a normal crossing support, where $X_{0}^{\prime}$ is the strict transform of $X_{0}$. We denote $\pi^{\prime}=\pi \circ \mu$. We set $\mu^{*} K_{X / S}=K_{Y / S}+\Gamma$. We have to prove that $-\Gamma$ is effective.

It is easy to see that $X$ is normal. The canonical divisor $K_{X}$ is known to be $\mathbb{Q}$ Cartier ([St, §6]). Thus what we have to prove is that any section of $\mathcal{O}_{X}\left(m K_{X / S}\right)$ for any positive integer $m$ can be lifted to a section of $\mathcal{O}_{Y}\left(m K_{Y / S}\right)$. Since the sections of $\mathcal{O}_{X_{0}}\left(m K_{X_{0}}\right)$ can be lifted to that of $\mathcal{O}_{X_{0}^{\prime}}\left(m K_{X_{0}^{\prime}}\right)$ by the assumption, the problem is reduced to extend sections of $\mathcal{O}_{X_{0}^{\prime}}\left(m K_{X_{0}^{\prime}}\right)$ to those of $\mathcal{O}_{Y}\left(m K_{Y / S}\right)$.

For positive integers $m$, we construct a series of projective birational morphisms $f_{m}: Y_{m} \rightarrow Y$ from smooth varieties which satisfy the following conditions (1) through (11). We denote $f_{m}^{\prime}=\mu \circ f_{m}$ and $\pi_{m}=\pi \circ f_{m}^{\prime}$. A divisor $\Delta_{m}$ on $Y_{m}$ is defined by $K_{Y_{m} / S}+\Delta_{m}=f_{m}^{*} K_{Y / S}$. We note that $-\Delta_{m}$ is effective.

(1) There is a decomposition

$$
f_{m}^{*}\left(m K_{Y / S}\right)=P_{m}+M_{m}
$$

in the group of divisors $\operatorname{Div}\left(Y_{m}\right)$.

(2) $P_{m}$ is $f_{m}^{\prime}$-free, i.e., the natural homomorphism $f_{m}^{\prime *} f_{m *}^{\prime} \mathcal{O}_{Y_{m}}\left(P_{m}\right) \rightarrow \mathcal{O}_{Y_{m}}\left(P_{m}\right)$ is surjective.

(3) $M_{m}$ is an effective divisor whose support is contained in a normal crossing divisor $F_{m}$.

(4) $M_{m}$ is the $f_{m}^{\prime}$-fixed part of $m K_{Y / S}$, that is, the natural homomorphism $f_{m *}^{\prime} \mathcal{O}_{Y_{m}}\left(P_{m}\right) \rightarrow \mu_{*} \mathcal{O}_{Y}\left(m K_{Y / S}\right)$ is bijective.

(5) The supports of the divisors $f_{m}^{*} Y_{0}, f_{m}^{*} \Gamma$ and $\Delta_{m}$ are contained in $F_{m}$.

For any very $\mu$-ample divisor $A$ on $Y$, there exist a positive integer $m_{0}$ and an effective divisor $B$ whose support does not contain $X_{0}^{\prime}$ such that $m_{0} K_{Y / S} \sim A+B$, because $X$ is a germ. By definition, we have $f_{m_{0}}^{*} B \geq M_{m_{0}}$.

(6) The support of the divisor $f_{m}^{*} B$ is contained in $F_{m}$.

We also assume that there is a projective birational morphism $g_{k m}: Y_{m} \rightarrow Y_{k}$ such that $f_{m}=f_{k} \circ g_{k m}$ for each pair of integers $k, m$ with $k<m$.

(7) The supports of $g_{k m}^{*} M_{k}$ and $g_{k m}^{*} F_{k}$ are contained in $F_{m}$.

We have $g_{k m}^{*} M_{k} / k \geq M_{m} / m$ if $k \mid m$. Let $Y_{m, 0}=f_{m}^{*} Y_{0}=X_{m, 0}^{\prime}+E_{m}$, where $X_{m, 0}^{\prime}$ is the strict transform of $X_{0}^{\prime}$. Let $Y_{m, 0}^{\prime}=f_{m}^{*} X_{0}^{\prime}$. Let $\mu_{0}: X_{0}^{\prime} \rightarrow X_{0}$, $f_{m, 0}: X_{m, 0}^{\prime} \rightarrow X_{0}^{\prime}, f_{m, 0}^{\prime}: X_{m, 0}^{\prime} \rightarrow X_{0}, \bar{f}_{m, 0}: Y_{m, 0}^{\prime} \rightarrow X_{0}^{\prime}, \bar{f}_{m, 0}^{\prime}: Y_{m, 0}^{\prime} \rightarrow X_{0}$, and $g_{k m, 0}: X_{m, 0}^{\prime} \rightarrow X_{k, 0}^{\prime}$ be the induced morphisms:

$$
\begin{aligned}
& Y_{m} \stackrel{g_{k m}}{\longrightarrow} Y_{k} \stackrel{f_{k}}{\longrightarrow} Y \stackrel{\mu}{\longrightarrow} X \stackrel{\pi}{\longrightarrow} S, \\
& X_{m, 0}^{\prime} \stackrel{g_{k m, 0}}{\longrightarrow} X_{k, 0}^{\prime} \stackrel{f_{k, 0}}{\longrightarrow} X_{0}^{\prime} \stackrel{\mu_{0}}{\longrightarrow} X_{0} .
\end{aligned}
$$

We define $\Gamma_{0}$ and $\Delta_{m, 0}$ by $K_{X_{0}^{\prime}}+\Gamma_{0}=\mu_{0}^{*} K_{X_{0}}$ and $K_{X_{m, 0}^{\prime}}+\Delta_{m, 0}=f_{m, 0}^{*} K_{X_{0}^{\prime}}$. Since $X_{0}$ has only canonical singularities, we have $\Gamma_{0} \leq 0$. We also have $\Delta_{m, 0} \leq 0$. By the adjunction formula, we have $\Gamma_{0}=\left.(\Gamma+E)\right|_{X_{0}^{\prime}}$ and

$$
\Delta_{m, 0}=\left.\left(\Delta_{m}+E_{m}-f_{m}^{*} E\right)\right|_{X_{m, 0}^{\prime}} .
$$

Let $B_{0}=\left.\left(B-m_{0} E\right)\right|_{X_{0}^{\prime}}, B_{m, 0}=\left.f_{m}^{*}\left(B-m_{0} E\right)\right|_{X_{m, 0}^{\prime}}$, and $F_{m, 0}=\left(F_{m}-X_{m, 0}^{\prime}\right) \cap$ $X_{m, 0}^{\prime}$. 
(8) There is another decomposition

$$
f_{m, 0}^{*}\left(m K_{X_{0}^{\prime}}\right)=Q_{m}+N_{m}
$$

in $\operatorname{Div}\left(X_{m, 0}^{\prime}\right)$.

(9) $Q_{m}$ is $f_{m, 0}^{\prime}$-free, i.e., the natural homomorphism $f_{m, 0}^{\prime *} f_{m, 0 *}^{\prime} \mathcal{O}_{X_{m, 0}^{\prime}}\left(Q_{m}\right) \rightarrow$ $\mathcal{O}_{X_{m, 0}^{\prime}}\left(Q_{m}\right)$ is surjective.

(10) $N_{m}$ is an effective divisor whose support is contained in the normal crossing divisor $F_{m, 0}$.

(11) $N_{m}$ is the $f_{m, 0}^{\prime}$-fixed part of $m K_{X_{0}^{\prime}}$, that is, the natural homomorphism $f_{m, 0 *}^{\prime} \mathcal{O}_{X_{m, 0}^{\prime}}\left(Q_{m}\right) \rightarrow \mu_{0 *} \mathcal{O}_{X_{0}^{\prime}}\left(m K_{X_{0}^{\prime}}\right)$ is bijective.

There is an injective homomorphism $\mu_{*} \mathcal{O}_{Y}\left(m K_{Y / S}\right) \rightarrow \mathcal{O}_{X}\left(m K_{X / S}\right)$, because $\mathcal{O}_{X}\left(m K_{X / S}\right)$ is a reflexive sheaf. Since $X_{0}$ has only canonical singularities, we have a natural homomorphism $\mathcal{O}_{X}\left(m K_{X / S}\right) \rightarrow \mu_{0 *} \mathcal{O}_{X_{0}^{\prime}}\left(m K_{X_{0}^{\prime}}\right)$ for $m \geq 0$. Therefore, there is a natural homomorphism

$$
\mu_{*} \mathcal{O}_{Y}\left(m K_{Y / S}\right) \rightarrow \mu_{0 *} \mathcal{O}_{X_{0}^{\prime}}\left(m K_{X_{0}^{\prime}}\right) .
$$

Thus we have $\left.P_{m}\right|_{X_{m, 0}^{\prime}} \leq Q_{m}$. We set

$$
M_{m, 0}=\left.\left(M_{m}-m f_{m}^{*} E\right)\right|_{X_{m, 0}^{\prime}} .
$$

Since $\left.\left(K_{Y / S}-E\right)\right|_{X_{0}^{\prime}}=K_{X_{0}^{\prime}}$, we have $M_{m, 0} / m \geq N_{m} / m$. We have $B_{m, 0} / m_{0} \geq$ $M_{m, 0} / m$ if $m_{0} \mid m$.

Definition 1. Let $f: Y \rightarrow X$ be a projective birational morphism of smooth varieties, and let $\pi: X \rightarrow S$ be another projective morphism to a base space $S$, which is assumed to be a quasi-projective variety or its germ at a point. Let $f^{\prime}=\pi \circ f$. Let $\Delta$ be a divisor on $Y$ defined by $K_{Y}+\Delta=f^{*} K_{X}$. Let $D$ be a divisor on $X$. Assume that $f^{*} D=P+N$, where $P$ is an $f^{\prime}$-nef and $f^{\prime}$-big $\mathbb{R}$-divisor, and $N$ is an effective $\mathbb{R}$-divisor whose support is a normal crossing divisor. The multiplier ideal sheaf $\mathcal{I}_{N}$ on $X$ is defined by the following formula: $\mathcal{I}_{N}=f_{*} \mathcal{O}_{Y}(\ulcorner-N-\Delta\urcorner)$. Since the effective part of the divisor $\ulcorner-N-\Delta\urcorner$ is exceptional for $f, \mathcal{I}_{N}$ is an ideal sheaf of $\mathcal{O}_{X}$ which is determined by $X$ and $N$. Alternatively, we have

$$
f_{*} \mathcal{O}_{Y}\left(\ulcorner P\urcorner+K_{Y}\right)=\mathcal{I}_{N}\left(D+K_{X}\right) .
$$

If $g: Y^{\prime} \rightarrow Y$ is a projective birational morphism from another smooth variety such that the support of $N^{\prime}=g^{*} N$ is a normal crossing divisor, then we have $\mathcal{I}_{N^{\prime}}=\mathcal{I}_{N}$, because $\left\ulcorner g^{*} P\right\urcorner+K_{Y^{\prime}} \geq g^{*}\left(\ulcorner P\urcorner+K_{Y}\right)$.

Since $R^{p} f_{*}^{\prime} \mathcal{O}_{Y}\left(\ulcorner P\urcorner+K_{Y}\right)=0$ and $R^{p} f_{*} \mathcal{O}_{Y}\left(\ulcorner P\urcorner+K_{Y}\right)=0$ for $p>0$, we have $R^{p} \pi_{*}\left(\mathcal{I}_{N}\left(D+K_{X}\right)\right)=0$ for $p>0$.

Proposition 2. Let $\pi: X \rightarrow S$ be a projective morphism from a smooth variety to a base space. Then there exists a $\pi$-ample divisor $A$ which depends only on $\pi$ such that the natural homomorphism $\pi^{*} \pi_{*}\left(\mathcal{I}_{N}\left(A+D+K_{X}\right)\right) \rightarrow \mathcal{I}_{N}\left(A+D+K_{X}\right)$ is surjective for any $f: Y \rightarrow X, D$ and $N$ as in Definition 1.

Proof. Let $x$ be any point of $X$, and $\mathfrak{m}_{x}$ the corresponding sheaf of ideals of $\mathcal{O}_{X}$. Let $g: X^{\prime} \rightarrow X$ be the blowing up at the ideal sheaf $\mathfrak{m}_{x}$. Let $E$ be the corresponding Cartier divisor on $X^{\prime}$; we have $\mathfrak{m}_{x} \mathcal{O}_{X^{\prime}}=\mathcal{O}_{X^{\prime}}(-E)$. We note that $-E$ is $g$-ample. Let $g^{\prime}=\pi \circ g$.

We take a $\pi$-ample divisor $A$ on $X$ such that the natural homomorphism

$$
g^{\prime *} g_{*}^{\prime}\left(\mathcal{O}_{X^{\prime}}\left(-E+g^{*} A\right)\right) \rightarrow \mathcal{O}_{X^{\prime}}\left(-E+g^{*} A\right)
$$


is surjective. Moreover, we assume that there is a surjective homomorphism $\mathcal{O}_{S}^{\oplus \ell} \rightarrow$ $g_{*}^{\prime} \mathcal{O}_{X^{\prime}}\left(-E+g^{*} A\right)$. Let $\mathcal{F}$ be the kernel of the induced homomorphism $\mathcal{O}_{X^{\prime}}^{\oplus \ell} \rightarrow$ $\mathcal{O}_{X^{\prime}}\left(-E+g^{*} A\right)$, which is a locally free sheaf on $X^{\prime}$. Let $V=\mathbb{P}(\mathcal{F})$ be the associated projective space bundle with the projection $\sigma: V \rightarrow X^{\prime}$ and the tautological sheaf $\mathcal{O}_{V}(H)$.

We may assume that there exists a projective birational morphism $h: Y \rightarrow X^{\prime}$ such that $f=g \circ h$. We set $W=\mathbb{P}\left(h^{*} \mathcal{F}\right)$. Let $\bar{h}: W \rightarrow V$ and $\tau: W \rightarrow Y$ be natural morphisms:

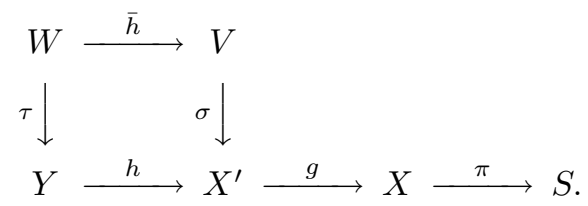

Let $\mathcal{J}$ be an ideal sheaf of $\mathcal{O}_{X^{\prime}}$ defined by $\mathcal{J}=h_{*} \mathcal{O}_{Y}(\ulcorner-N-\Delta\urcorner)$. Then we have $h_{*} \mathcal{O}_{Y}\left(\ulcorner P\urcorner+K_{Y}\right)=\mathcal{J}\left(g^{*}\left(D+K_{X}\right)\right)$ and $g_{*} \mathcal{J}=\mathcal{I}_{N}$. We take a positive integer $b$ such that the divisor $H-b \sigma^{*} E-K_{V / X^{\prime}}$ on $V$ is $(g \circ \sigma)$-ample. We also assume now that the divisor $-(b+1) E+g^{*} A$ is $g^{\prime}$-ample. Then we have for $p>0$

$$
\begin{aligned}
& R^{p} f_{*}\left(h^{*} \mathcal{F}\left(-b h^{*} E+\ulcorner P\urcorner+K_{Y}\right)\right) \\
& =R^{p}(f \circ \tau)_{*} \mathcal{O}_{W}\left(\bar{h}^{*}\left(H-b \sigma^{*} E-K_{V / X^{\prime}}\right)+\left\ulcorner\tau^{*} P\right\urcorner+K_{W}\right)=0, \\
& R^{p} h_{*}\left(h^{*} \mathcal{F}\left(-b h^{*} E+\ulcorner P\urcorner+K_{Y}\right)\right)=0,
\end{aligned}
$$

hence

$$
R^{1} g_{*}(\mathcal{J} \otimes \mathcal{F}(-b E))=0
$$

Similarly, we have

$$
R^{1} g_{*}^{\prime}\left(\mathcal{J}\left(-(b+1) E+g^{*}\left(A+D+K_{X}\right)\right)\right)=R^{1} g_{*}(\mathcal{J}(-(b+1) E))=0 .
$$

Therefore, from an exact sequence

$$
0 \rightarrow \mathcal{J} \otimes \mathcal{F}(-b E) \rightarrow \mathcal{J}^{\oplus \ell}(-b E) \rightarrow \mathcal{J}\left(-(b+1) E+g^{*} A\right) \rightarrow 0,
$$

we obtain an exact sequence

$$
0 \rightarrow g_{*}(\mathcal{J} \otimes \mathcal{F}(-b E)) \rightarrow g_{*}\left(\mathcal{J}^{\oplus \ell}(-b E)\right) \rightarrow g_{*}\left(\mathcal{J}\left(-(b+1) E+g^{*} A\right)\right) \rightarrow 0 .
$$

Thus

$$
g_{*}(\mathcal{J}(-(b+1) E))=g_{*}(\mathcal{J}(-b E)) \cdot \mathfrak{m}_{x} \subset \mathcal{I}_{N} \cdot \mathfrak{m}_{x} .
$$

On the other hand, we have surjective homomorphisms

$$
\begin{aligned}
& g_{*}^{\prime}\left(\mathcal{J}\left(g^{*}\left(A+D+K_{X}\right)\right)\right) \rightarrow g_{*}^{\prime}\left(\mathcal{J}\left(g^{*}\left(A+D+K_{X}\right)\right) \otimes \mathcal{O}_{(b+1) E}\right), \\
& g_{*}\left(\mathcal{J}\left(g^{*}\left(A+D+K_{X}\right)\right)\right) \rightarrow g_{*}\left(\mathcal{J}\left(g^{*}\left(A+D+K_{X}\right)\right) \otimes \mathcal{O}_{(b+1) E}\right)
\end{aligned}
$$

whose images are naturally identified. Hence we obtain natural surjective homomorphisms

$$
\begin{aligned}
& \pi_{*}\left(\mathcal{I}_{N}\left(A+D+K_{X}\right)\right) \\
& \rightarrow g_{*}\left(\mathcal{J}\left(g^{*}\left(A+D+K_{X}\right)\right)\right) / g_{*}\left(\mathcal{J}\left(-(b+1) E+g^{*}\left(A+D+K_{X}\right)\right)\right) \\
& \rightarrow \mathcal{I}_{N}\left(A+D+K_{X}\right) \otimes \mathcal{O}_{X} / \mathfrak{m}_{x}
\end{aligned}
$$


Remark 3. The above proof can be easily extended to the case in which $X$ is singular. As long as $X$ is smooth, one can use the Koszul resolution of $\mathcal{O}_{X^{\prime}}(-E)$ instead of the sheaf $\mathcal{F}$ of the above proof in order to obtain a more precise condition on the divisor $A$.

Since $\left.K_{Y / S}\right|_{X_{0}^{\prime}}=K_{X_{0}^{\prime}}+\left.E\right|_{X_{0}^{\prime}}$, we have

$$
\begin{aligned}
f_{m *} \mathcal{O}_{Y_{m}}\left(\left\ulcorner k P_{m} / m\right\urcorner+K_{Y_{m} / S}\right) & =\mathcal{I}_{k M_{m} / m}\left((k+1) K_{Y / S}\right), \\
f_{m, 0 *} \mathcal{O}_{X_{m, 0}^{\prime}}\left(\left\ulcorner k P_{m} /\left.m\right|_{X_{m, 0}^{\prime}}\right\urcorner+K_{X_{m, 0}^{\prime}}\right) & =\mathcal{I}_{k M_{m, 0} / m}\left((k+1) K_{X_{0}^{\prime}}\right), \\
f_{m, 0 *} \mathcal{O}_{X_{m, 0}^{\prime}}\left(\left\ulcorner k Q_{m} / m\right\urcorner+K_{X_{m, 0}^{\prime}}\right) & =\mathcal{I}_{k N_{m} / m}\left((k+1) K_{X_{0}^{\prime}}\right) .
\end{aligned}
$$

We note that the support of $\mathcal{O}_{Y} / \mathcal{I}_{k M_{m} / m}$ does not contain $X_{0}^{\prime}$.

Since $g_{m m^{\prime}, 0}^{*} N_{m} / m \geq N_{m^{\prime}} / m^{\prime}$ if $m \mid m^{\prime}$, we have $\mathcal{I}_{k N_{m} / m} \subset \mathcal{I}_{k N_{m^{\prime}} / m^{\prime}}$ for any positive integer $k$. We define

$$
\mathcal{I}_{k N}=\bigcup_{m=1}^{\infty} \mathcal{I}_{k N_{m} / m}
$$

Since $\mathcal{O}_{X_{0}^{\prime}}$ is noetherian, there exists a positive integer $m$, which depends on $k$, such that $\mathcal{I}_{k N}=\mathcal{I}_{k N_{m} / m}$. Similarly, we define $\mathcal{I}_{k M}=\bigcup_{m=1}^{\infty} \mathcal{I}_{k M_{m, 0} / m}$ and $\mathcal{I}_{k \bar{M}}=$ $\bigcup_{m=1}^{\infty} \mathcal{I}_{k M_{m} / m}$.

\section{Lemma 4.}

$$
\begin{aligned}
& \operatorname{Im}\left(\mu_{*} \mathcal{O}_{Y}\left((k+1) K_{Y / S}\right) \rightarrow \mu_{0 *} \mathcal{O}_{X_{0}^{\prime}}\left((k+1) K_{X_{0}^{\prime}}\right)\right) \\
& =\mu_{0 *}\left(\mathcal{I}_{k M}\left((k+1) K_{X_{0}^{\prime}}\right)\right) \\
& =\mu_{0 *}\left(\mathcal{I}_{(k+1) M}\left((k+1) K_{X_{0}^{\prime}}\right)\right) .
\end{aligned}
$$

Proof. Since $\mathcal{I}_{(k+1) M} \subset \mathcal{I}_{k M}$, we have

$$
\mu_{0 *}\left(\mathcal{I}_{(k+1) M}\left((k+1) K_{X_{0}^{\prime}}\right)\right) \subset \mu_{0 *}\left(\mathcal{I}_{k M}\left((k+1) K_{X_{0}^{\prime}}\right)\right) .
$$

If $s \in \mu_{0 *} \mathcal{O}_{X_{0}^{\prime}}\left((k+1) K_{X_{0}^{\prime}}\right)$ is the image of $\bar{s} \in \mu_{*} \mathcal{O}_{Y}\left((k+1) K_{Y / S}\right)$, then

$$
\operatorname{div}\left(f_{k+1}^{*} \bar{s}\right) \geq M_{k+1} \quad \text { on } Y_{k+1} .
$$

Since $\left.K_{Y / S}\right|_{X_{0}^{\prime}}=K_{X_{0}^{\prime}}+\left.E\right|_{X_{0}^{\prime}}$ and $M_{k+1,0}=\left.\left(M_{k+1}-(k+1) f_{k+1}^{*} E\right)\right|_{X_{k+1,0}^{\prime}}$, we have $\operatorname{div}\left(f_{k+1,0}^{*} s\right) \geq M_{k+1,0}$, hence $s \in \mu_{0 *} I_{M_{k+1,0}}\left((k+1) K_{X_{0}}\right)$. Since $I_{M_{k+1,0}} \subset I_{(k+1) M}$, we have

$$
\operatorname{Im}\left(\mu_{*} \mathcal{O}_{Y}\left((k+1) K_{Y / S}\right) \rightarrow \mu_{0 *} \mathcal{O}_{X_{0}^{\prime}}\left((k+1) K_{X_{0}^{\prime}}\right)\right) \subset \mu_{0 *}\left(\mathcal{I}_{(k+1) M}\left((k+1) K_{X_{0}^{\prime}}\right)\right) .
$$

We shall prove that

$$
\mu_{0 *}\left(\mathcal{I}_{k M}\left((k+1) K_{X_{0}^{\prime}}\right)\right) \subset \operatorname{Im}\left(\mu_{*} \mathcal{O}_{Y}\left((k+1) K_{Y / S}\right) \rightarrow \mu_{0 *} \mathcal{O}_{X_{0}^{\prime}}\left((k+1) K_{X_{0}^{\prime}}\right)\right) .
$$

We take a positive integer $m$ such that $\mathcal{I}_{k M}=\mathcal{I}_{k M_{m, 0} / m}$ and $\mathcal{I}_{k \bar{M}}=\mathcal{I}_{k M_{m} / m}$. Since $R^{1} f_{m *} \mathcal{O}_{Y_{m}}\left(\left\ulcorner k P_{m} / m\right\urcorner+K_{Y_{m} / S}\right)=0$, we obtain an exact sequence

$$
\begin{aligned}
0 & \rightarrow f_{m *} \mathcal{O}_{Y_{m}}\left(\left\ulcorner k P_{m} / m\right\urcorner+K_{Y_{m} / S}-Y_{m, 0}^{\prime}\right) \rightarrow f_{m *} \mathcal{O}_{Y_{m}}\left(\left\ulcorner k P_{m} / m\right\urcorner+K_{Y_{m} / S}\right) \\
& \rightarrow \bar{f}_{m, 0 *}\left(\mathcal{O}_{Y_{m}}\left(\left\ulcorner k P_{m} / m\right\urcorner+K_{Y_{m} / S}\right) \otimes \mathcal{O}_{Y_{m, 0}^{\prime}}\right) \rightarrow 0 .
\end{aligned}
$$

On the other hand, since $\mathcal{O}_{Y_{m}}\left(K_{Y_{m} / S}\right) \otimes \mathcal{O}_{Y_{m, 0}^{\prime}}=\omega_{Y_{m, 0}^{\prime}}\left(f_{m}^{*} E\right)$, there is a natural injective homomorphism

$$
\begin{aligned}
& f_{m, 0 *} \mathcal{O}_{X_{m, 0}^{\prime}}\left(\left\ulcorner k P_{m} /\left.m\right|_{X_{m, 0}^{\prime}}\right\urcorner+K_{X_{m, 0}^{\prime}}\right) \\
& \quad \rightarrow \bar{f}_{m, 0 *}\left(\mathcal{O}_{Y_{m}}\left(\left\ulcorner k P_{m} / m\right\urcorner+K_{Y_{m} / S}\right) \otimes \mathcal{O}_{Y_{m, 0}^{\prime}}\right)(-E) .
\end{aligned}
$$


Therefore, there is an injective homomorphism

$$
\mathcal{I}_{k M}\left((k+1) K_{X_{0}^{\prime}}\right) \rightarrow \mathcal{I}_{k \bar{M}}\left((k+1) K_{Y / S}\right) \otimes \mathcal{O}_{X_{0}^{\prime}}(-E) .
$$

Since $R^{1} \mu_{*}\left(\mathcal{I}_{k \bar{M}}\left((k+1) K_{Y / S}\right)\right)=0$, the homomorphism

$$
\mu_{*}\left(\mathcal{I}_{k \bar{M}}\left((k+1) K_{Y / S}\right)\right) \rightarrow \mu_{*}\left(\mathcal{I}_{k \bar{M}}\left((k+1) K_{Y / S}\right) \otimes \mathcal{O}_{Y_{0}}\right)
$$

is surjective. On the other hand, since the natural homomorphism

$$
\mu_{*} \mathcal{I}_{M_{k+1}}\left((k+1) K_{Y / S}\right) \rightarrow \mu_{*} \mathcal{O}_{Y}\left((k+1) K_{Y / S}\right)
$$

is bijective, so is $\mu_{*} \mathcal{I}_{k \bar{M}}\left((k+1) K_{Y / S}\right) \rightarrow \mu_{*} \mathcal{O}_{Y}\left((k+1) K_{Y / S}\right)$. Since we have an injective homomorphism $\mathcal{O}_{X_{0}^{\prime}}(-E) \rightarrow \mathcal{O}_{Y_{0}}$, the lemma is proved.

Lemma 5. Assume that there exists a divisor $C$ on $X_{0}^{\prime}$ such that $\mathcal{I}_{k N}(-C) \subset \mathcal{I}_{k M}$ for all positive integers $k$. Then the natural homomorphism $\mu_{0 *}\left(\mathcal{I}_{k M}\left(k K_{X_{0}^{\prime}}\right)\right) \rightarrow$ $\mu_{0 *} \mathcal{O}_{X_{0}^{\prime}}\left(k K_{X_{0}^{\prime}}\right)$ is bijective for any positive integer $k$.

Proof. If the lemma does not hold, then there exists a positive integer $k$ such that some of the coefficients of

$$
g_{k m, 0}^{*} N_{k}-k M_{m, 0} / m-\Delta_{m, 0}+F_{m, 0}
$$

at the irreducible components of $F_{m, 0}$ are not positive for any positive integer $m$ with $m \geq k$. Indeed, if $\left\ulcorner-k M_{m, 0} / m\right\urcorner-\Delta_{m, 0} \geq-g_{k m, 0}^{*} N_{k}$, then the homomorphism $\mu_{0 *}\left(\mathcal{I}_{k M}\left(k K_{X_{0}^{\prime}}\right)\right) \rightarrow \mu_{0 *} \mathcal{O}_{X_{0}^{\prime}}\left(k K_{X_{0}^{\prime}}\right)$ is surjective.

Since $\mathcal{I}_{k^{\prime} N}(-C) \subset \mathcal{I}_{k^{\prime} M}$ for any $k^{\prime}$, there exist positive integers $m^{\prime}$, depending on $k^{\prime}$, such that

$$
g_{k^{\prime} m^{\prime}, 0}^{*} N_{k^{\prime}}+f_{m^{\prime}, 0}^{*} C-k^{\prime} M_{m^{\prime}, 0} / m^{\prime}-\Delta_{m^{\prime}, 0}+F_{m^{\prime}, 0}
$$

has strictly positive coefficients at the irreducible components of $F_{m^{\prime}, 0}$, because $\mathcal{O}_{X_{k^{\prime}, 0}^{\prime}}\left(-N_{k^{\prime}}\right) \subset \mathcal{I}_{k^{\prime} N} \mathcal{O}_{X_{k^{\prime}, 0}^{\prime}}$. Since $g_{k k^{\prime}, 0}^{*} N_{k} / k \geq N_{k^{\prime}} / k^{\prime}$ if $k \mid k^{\prime}$, the coefficients of

$$
\begin{aligned}
& g_{k m^{\prime}, 0}^{*} N_{k}-k M_{m^{\prime}, 0} / m^{\prime}-\Delta_{m^{\prime}, 0}+F_{m^{\prime}, 0} \\
& +\frac{k}{k^{\prime}}\left(f_{m^{\prime}, 0}^{*} C+\frac{k^{\prime}-k}{k} \Delta_{m^{\prime}, 0}-\frac{k^{\prime}-k}{k} F_{m^{\prime}, 0}\right)
\end{aligned}
$$

are positive.

We may assume that the support of $f_{k, 0}^{*} C$ is contained in $F_{k, 0}$. Then

$$
-f_{k, 0}^{*} C-\frac{k^{\prime}-k}{k} \Delta_{k, 0}+\frac{k^{\prime}-k}{k} F_{k, 0}
$$

is effective for sufficiently large $k^{\prime}$. Since $g_{k m^{\prime}, 0}^{*}\left(-\Delta_{k, 0}+F_{k, 0}\right) \leq-\Delta_{m^{\prime}, 0}+F_{m^{\prime}, 0}$ for $k \leq m^{\prime}$, it follows that

$$
-f_{m^{\prime}, 0}^{*} C-\frac{k^{\prime}-k}{k} \Delta_{m^{\prime}, 0}+\frac{k^{\prime}-k}{k} F_{m^{\prime}, 0}
$$

is also effective for such $k^{\prime}$ and for any $m^{\prime} \geq k$. But this is a contradiction.

Proof of the Main Theorem. We have to prove that the natural homomorphism

$$
\mu_{*} \mathcal{O}_{Y}\left(m K_{Y / S}\right) \rightarrow \mu_{0 *} \mathcal{O}_{X_{0}^{\prime}}\left(m K_{X_{0}^{\prime}}\right)
$$

is surjective for any positive integer $m$. Suppose the contrary. Then there exists a positive integer $k$ such that $\mu_{0 *}\left(\mathcal{I}_{k M}\left(k K_{X_{0}^{\prime}}\right)\right) \neq \mu_{0 *} \mathcal{O}_{X_{0}^{\prime}}\left(k K_{X_{0}^{\prime}}\right)$ by Lemma 4 .

We consider the conditions

$$
\mathcal{I}_{(k-1) N}\left(-B_{0}\right) \subset \mathcal{I}_{\left(k+m_{0}-1\right) M}
$$


for positive integers $k$. For $k=1$, the condition is satisfied, because $\mathcal{O}_{X_{0}^{\prime}}\left(-B_{0}\right) \subset$ $\mathcal{I}_{m_{0} M}$. By Lemma 5 , there exists a positive integer $k$ such that $\mathcal{I}_{(k-1) N}\left(-B_{0}\right) \subset$ $\mathcal{I}_{\left(k+m_{0}-1\right) M}$ but $\mathcal{I}_{k N}\left(-B_{0}\right) \not \subset \mathcal{I}_{\left(k+m_{0}\right) M}$.

We take $A$ such that $A_{0}-K_{X_{0}^{\prime}}$ for $A_{0}=\left.A\right|_{X_{0}^{\prime}}$ satisfies Proposition 2 for $\mu_{0}$ : $X_{0}^{\prime} \rightarrow X_{0}$. Since $A_{0}+B_{0} \sim m_{0} K_{X_{0}^{\prime}}$, we have

$$
\mathcal{I}_{k N}\left(-B_{0}+\left(k+m_{0}\right) K_{X_{0}^{\prime}}\right) \simeq \mathcal{I}_{k N}\left(A_{0}-K_{X_{0}^{\prime}}+(k+1) K_{X_{0}^{\prime}}\right) .
$$

Thus the homomorphism

$$
\mu_{0}^{*} \mu_{0 *}\left(\mathcal{I}_{k N}\left(-B_{0}+\left(k+m_{0}\right) K_{X_{0}^{\prime}}\right)\right) \rightarrow \mathcal{I}_{k N}\left(-B_{0}+\left(k+m_{0}\right) K_{X_{0}^{\prime}}\right)
$$

is surjective by Proposition 2 .

By Lemma 4, we have

$$
\begin{aligned}
& \mu_{0 *}\left(\mathcal{I}_{k N}\left(-B_{0}+\left(k+m_{0}\right) K_{X_{0}^{\prime}}\right)\right) \\
& \subset \mu_{0 *}\left(\mathcal{I}_{(k-1) N}\left(-B_{0}+\left(k+m_{0}\right) K_{X_{0}^{\prime}}\right)\right) \\
& \subset \mu_{0 *}\left(\mathcal{I}_{\left(k+m_{0}-1\right) M}\left(\left(k+m_{0}\right) K_{X_{0}^{\prime}}\right)\right) \\
& =\mu_{0 *}\left(\mathcal{I}_{\left(k+m_{0}\right) M}\left(\left(k+m_{0}\right) K_{X_{0}^{\prime}}\right)\right) .
\end{aligned}
$$

But

$$
\mathcal{I}_{k N}\left(-B_{0}+\left(k+m_{0}\right) K_{X_{0}^{\prime}}\right) \not \subset \mathcal{I}_{\left(k+m_{0}\right) M}\left(\left(k+m_{0}\right) K_{X_{0}^{\prime}}\right),
$$

a contradiction.

The following is a slight generalization of a theorem of Siu [Si]:

Theorem 6. Let $\pi: X \rightarrow S$ be a projective flat morphism from a normal variety to a germ of a smooth curve $\left(S, s_{0}\right)$. Assume that the fibers $X_{s}=\pi^{-1}(s)$ have only canonical singularities and are of general type for all $s \in S$. Then the plurigenus $P_{m}\left(X_{s}\right)=\operatorname{dim} H^{0}\left(X_{s}, m K_{X_{s}}\right)$ is constant as a function on $s \in S$ for any positive integer $m$.

Proof. The proof is very similar to that of the Main Theorem. We only point out how to modify the proof. First, the morphisms $f_{m}: Y_{m} \rightarrow Y$ are constructed only for those $m$ such that $P_{m}\left(X_{s}\right) \neq 0$ for general fibers $X_{s}$. In the conditions (2) and (9), $P_{m}$ and $Q_{m}$ are $\pi_{m}$-free and free instead of $f_{m}^{\prime}$-free and $f_{m, 0}^{\prime}$-free, respectively. In the conditions (4) and (11), $M_{m}$ and $N_{m}$ are the $\pi_{m}$-fixed part and the fixed part instead of the $f_{m}^{\prime}$-fixed part and the $f_{m, 0}^{\prime}$-fixed part, respectively. The divisor $A$ is very $\pi^{\prime}$-ample instead of very $\mu$-ample. The reason for Supp $B \not \supset X_{0}^{\prime}$ is the following: since $-\Gamma$ is effective, it follows otherwise that $B \geq Y_{0}$, and we can replace $B$ by a smaller divisor. The homomorphism in the formula before Definition 1 is replaced by the homomorphism $\pi_{*}^{\prime} \mathcal{O}_{Y}\left(m K_{Y / S}\right) \rightarrow H^{0}\left(X_{0}^{\prime}, m K_{X_{0}^{\prime}}\right)$.

In the definitions of $\mathcal{I}_{k N}, \mathcal{I}_{k M}$ and $\mathcal{I}_{k \bar{M}}$, the union is taken over those $m$ which we considered. In Lemmas 4 and 5, their proofs and in the proof of the Main Theorem, $\mu_{*}$ and $\mu_{0 *}$ are replaced by $\pi_{*}^{\prime}$ and $H^{0}$. In the proof of Lemma 5 , we take $k$ such that $P_{k}\left(X_{s_{0}}\right) \neq 0$, and we assume that $k \mid k^{\prime}$. In the proof of the Main Theorem, the morphism $\mu_{0}$ is replaced by the structure morphism $X_{0}^{\prime} \rightarrow$ Spec $\mathbb{C}$. The homomorphism in the formula in the middle of the proof of the Main Theorem is replaced by the statement that the sheaf $\mathcal{I}_{k N}\left(-B_{0}+\left(k+m_{0}\right) K_{X_{0}^{\prime}}\right)$ is generated by global sections. 


\section{REFERENCES}

[I] S. Ishii, Small deformations of normal singularities, Math. Ann. 275 (1986), 139-148. MR 87i:14003; MR 88c:14004

$[\mathrm{KMM}]$ Y. Kawamata, K. Matsuda and K. Matsuki, Introduction to the minimal model problem, Adv. St. Pure Math. 10 (1987), 283-360. MR 89e:14015

[Si] Y.-T. Siu, Invariance of plurigenera, preprint, 1997.

[St] J. Stevens, On canonical singularities as total spaces of deformations, Abh. Math. Sem. Univ. Hamburg 58 (1988), 275-283. MR 91c:14007

Department of Mathematical Sciences, University of Tokyo, Komaba, Meguro, Tokyo, 153-8914, JAPAN

E-mail address: kawamata@ms.u-tokyo.ac.jp 